(C) 2006 IEEE. Personal use of this material is permitted. However, permission to reprint/republish this material for advertising or promotional purposes or for creating new collective works for resale or redistribution to servers or lists, or to reuse any copyrighted component of this work in other works must be obtained from the IEEE. 
[15] M. Meila and J. Shi, "Learning segmentation by random walks," in Advances in Neural Information Processing Systems 13, T. K. Leen, T. G. Dietterich, and V. Tresp, Eds. Cambridge, MA: MIT Press, 2001, pp. 873-879.

[16] J. Larsen, L. Hansen, A. Szymkowiak-Have, T. Christiansen, and T. Kolenda, "Webmining: Learning from the world wide web," Special Issue of Computat. Statist. Data Anal., vol. 38, pp. 517-532, 2002.

[17] J. Larsen, A. Szymkowiak-Have, and L. Hansen, "Probabilistic hierarchical clustering with labeled and unlabeled data," Int. J. KnowledgeBased Intell. Eng. Syst., vol. 6, no. 1, pp. 56-62, 2002.

[18] A. Szymkowiak, J. Larsen, and L. Hansen, "Hierarchical clustering for datamining," in Proc. KES-2001 5th Int. Conf. Knowledge-Based Intelligent Info. Eng. Syst. Allied Technol., 2001, pp. 261-265.

\section{On Stability of Recurrent Neural Networks-An Approach From Volterra Integro-Differential Equations}

\author{
Pingzhou Liu and Qing-Long Han
}

\begin{abstract}
The uniform asymptotic stability of recurrent neural networks (RNNs) with distributed delay is analyzed by comparing RNNs to linear Volterra integro-differential systems under Lipschitz continuity of activation functions. The stability criteria obtained have unified and extended many existing results on RNNs.
\end{abstract}

Index Terms-Delay, recurrent neural networks (RNNs), stability, Volterra integro-differential systems.

\section{INTRODUCTION}

In this letter, we consider the following recurrent neural network (RNN) model

$$
\left\{\begin{array}{l}
\frac{d x(t)}{d t}=-D x(t)+B \int_{a}^{t} K(t-s) F(x(s)) d s \\
x(s)=\phi(s), \quad x \in\left(a, t_{0}\right), \quad t_{0} \geq 0
\end{array}\right.
$$

which is the generalization of the most extensively studied model

$$
\frac{d y}{d t}=-D y+B F(y)+D u . \quad y\left(t_{0}\right)=y_{0}
$$

by introducing distributed delay and translating equilibrium to the origin, where $x, y \in R^{n}$ are the state vectors, $D=$ $\operatorname{diag}\left(d_{1}, d_{2}, \ldots, d_{n}\right) \in R^{n \times n}$ is a constant diagonal matrix with $d_{i}>0, B=\left[b_{i j}\right] \in R^{n \times n}$ is a constant connection weight matrix, $u \in R^{n}$ is a constant input vector, the delay kernel $K(\cdot)=\left[k_{i j}(\cdot)\right] \in L^{1}\left(R^{+}\right) . F(\cdot)=\operatorname{col}\left(f_{1}(\cdot), f_{2}(\cdot), \ldots, f_{n}(\cdot)\right)$

Manuscript received March 21, 2004; revised July 25, 2005. This work was supported in part by the Faculty of Informatics and Communication, Central Queensland University, for the 2003-2004 Research Project "A Volterra integro-differential equation approach to stability for a class of recurrent neural networks."

P. Liu is with the Faculty of Informatics and Communication, Central Queensland University, Rockhampton, Australia (e-mail: q.han@cqu.edu.au), on leave from Flinders University, South Australia, Australia, and Shanxi Normal University, China.

Q.-L. Han is with the Faculty of Informatics and Communication, Central Queensland University, Rockhampton, Australia.

Digital Object Identifier 10.1109/TNN.2005.860859 is a vector-value activation function from $R^{n}$ to $R^{n}$ and is assumed to be of class $\mathcal{G} \mathcal{L}$ or $\mathcal{L}$ and $F(0)=0$. If $F \in \mathcal{G L}$, then there exist $\ell_{i}$, such that $\forall x, y \in R$ and $x \neq y$

$$
0 \leq \frac{f_{i}(x)-f_{i}(y)}{x-y} \leq \ell_{i}, \quad i=1,2, \ldots, n
$$

If $F \in \mathcal{L}$, then there exist constants $\ell_{i}$, such that $\forall x, y \in R$

$$
\left|f_{i}(x)-f_{i}(y)\right| \leq \ell_{i}|x-y|, \quad i=1,2, \ldots, n .
$$

In the following we only consider the case of $a=0$ or $a=-\infty$. If $a=0$, the system has finite memory and one needs to deal with "uniform" stability. If $a=-\infty$, the system has infinite memory. In practice situations, the distant past usually has less influence compared to the recent behavior of the state. The case of $a=-\infty$, which has drawn the most concern in the neural networks research, is just a mathematical simplification.

In this letter, we will use the well-known results about linear Volterra integro-differential equations and the nonlinearity nature of Lipschitz continuity (3) or (4), to study the global uniform asymptotic stability of (1). As we put different kinds of delays under one umbrella-distributed delay, it also provides a way to approximately consider the delay dependency of the neural networks by choosing an appropriate and easy-to-handle delay kernel.

\section{VOLTERRA INTEGRO-DIFFERENTIAL EQUATIONS}

Let $C(a, \infty)(a=0$ or $a=-\infty)$ denote the set of all continuous functions $\varphi:(a, \infty) \rightarrow R^{n}$ such that, for any $t \in R^{1}$, the semi-norm

$$
\|\varphi\|_{t}=\sup \{|\varphi(s)|: a<s \leq t\}
$$

is finite. Let $B(\cdot) \in L^{1}\left(R^{+}\right)$be a real matrix function.

Consider the following linear Volterra integro-differential equation [9], [10]

$$
\frac{d x(t)}{d t}=A x(t)+\int_{a}^{t} B(t-s) x(s) d s
$$

for $t \geq t_{0}$ with $x(t)=\varphi(t)$, where $x(t)=$ $\operatorname{col}\left(x_{1}(t), x_{2}(t), \ldots, x_{n}(t)\right) \in R^{n}$ with Euclidean norm $|x|=\left(\sum_{i=1}^{n} x_{i}^{2}\right)^{1 / 2}$ and $A$ is a real constant matrix, $t \geq t_{0}$ and $x(t)=\phi(t)$ on $a \leq t \leq t_{0}$. The solution of (5) with initial values $\left(t_{0}, \phi\right)$ will be denoted by $x\left(t, t_{0}, \phi\right)$.

Notice that for $a=-\infty$, (5) is autonomous. It follows that one needs only to consider the case of $a=-\infty$ with initial time $t_{0}=0$. Moreover, stability and uniform stability are equivalent.

Let

$$
\begin{aligned}
\beta_{i j} & =\int_{0}^{\infty} b_{i j}(t) d t, \quad \beta_{i j}^{+}=\int_{0}^{\infty}\left|b_{i j}(t)\right| d t \quad \text { and } \\
R_{i} & =\sum_{j=1}^{n}\left(\left|a_{i j}\right|+\beta_{i j}{ }^{+}\right) .
\end{aligned}
$$

Theorem 1: [9]: Let $B(\cdot) \in L^{1}$ and

$$
\left|a_{i i}+\beta_{i i}\right|\left|a_{k k}+\beta_{k k}\right|>\left(\sum_{j \neq i}\left|a_{i j}+\beta_{i j}\right|\right)\left(\sum_{j \neq k}\left|a_{k j}+\beta_{k j}\right|\right)
$$


for all $i \neq k$, and $i, k=1,2, \ldots, n$. Then the trivial solution of (5) is uniformly asymptotically stable if for each $i=1, \ldots, n$, either of the following conditions holds:

i) $a_{i i}<0$ and $\left|a_{i i}\right| \geq \sum_{j \neq i}\left|a_{i j}\right|+\sum_{j=1}^{n} \beta_{i j}{ }^{+}$;

ii) $a_{i i}+\beta_{i i}<0, T_{i}=\int_{0}^{\infty} t\left|b_{i i}(t)\right| d t<\infty$ and

$$
T_{i} \leq\left(\left|a_{i i}+\beta_{i i}\right|-\sum_{j \neq i}\left(\left|a_{i j}\right|+\beta_{i j}{ }^{+}\right)\right) / R_{i} .
$$

To generalize the condition (i) of Theorem 1 , we use $M$-matrix theory to derive another sufficient condition which is weaker than (i) but also guarantees the uniform asymptotic stability of the trivial solution of (5).

Using the properties of $M$-matrix and letting $c_{i i}=a_{i i}+\beta_{i i}{ }^{+}, c_{i j}=$ $\left|a_{i j}\right|+\beta_{i j}{ }^{+}, i \neq j$, we can prove the following result.

Proposition 1: If $B(\cdot) \in L^{1}\left(R^{+}\right)$and $-C=\left(-c_{i j}\right)$ is an $M$-matrix, then the trivial solution of (5) is uniformly asymptotically stable.

Proof: Noting that $c_{i j}>0(i \neq j)$, the matrix $-C$ has nonpositive off-diagonal elements; it is a possible candidate of an $M$-matrix. From the properties of $M$-matrix, there exists a diagonal matrix $P=\operatorname{diag}\left(p_{1}, p_{2}, \ldots p_{n}\right),\left(p_{i}>0\right)$, such that $-C P$ is diagonally dominant, that is

$$
-c_{i i}>0 \text { and } \quad-c_{i i} p_{i}>\sum_{j \neq i} c_{i j} p_{j} .
$$

As $\beta_{i i}{ }^{+}>0$, the following hold

$$
a_{i i}<0 \text { and }\left|a_{i i}\right| p_{i}>\sum_{j \neq i}\left|a_{i j}\right| p_{j}+\sum_{j} \beta_{i j}{ }^{+} p_{j} .
$$

Let $x(t)$ be a solution of (5) and $y(t)=P^{-1} x(t)$. Then $y(t)$ satisfies

$$
\frac{d y(t)}{d t}=P^{-1} A P y(t)+\int_{a}^{t} P^{-1} B(t-s) P y(s) d s .
$$

Clearly, the stability properties of (5) and (8) are equivalent. Following the above inequalities from $M$-matrix that (8) satisfies the condition (i) of Theorem 1. It is easy to prove the condition (6) in Theorem 1 for (8) is true from $\beta_{i j}^{+} \geq\left|\beta_{i j}\right|$ and a simple estimation by using the strict inequality of (7). Then the conclusion follows.

We aim to use the results about Volterra integral-differential equations to neural networks where nonlinearity is common. In the remaining part of this section we will consider a class of nonlinear Volterra-type integro-differential equations

$$
\begin{cases}\frac{d u(t)}{d t}=A u(t)+F\left(\int_{a}^{t} B(t-s) u(s) d s\right) & t \geq t_{0} \geq 0 \\ u(t)=\phi(t) & \text { for } t \leq t_{0}\end{cases}
$$

where $F: C(R) \rightarrow R^{n}$ satisfies $F(0)=0$ and condition (4), under which it can be proved that (9) has a global solution [8].

Now, consider the linear Volterra system

$$
\frac{d x(t)}{d t}=A x(t)+\int_{a}^{t} L B(t-s) x(s) d s
$$

with the same initial values as (9), where $L$ is the Lipschitz constant of $F$.

Proposition 2: If the trivial solution of (10) is uniformly asymptotically stable, then the trivial solution of (9) is uniformly stable. If, in addition, $F(\cdot) \in \mathcal{L}$ and the conditions (i) of Theorem 1 or Proposition 1 to (10) hold, or $F(\cdot) \in \mathcal{G L}$ and the condition (ii) of Theorem 1 to (10) holds, then the trivial solution of (9) is globally uniformly asymptotically stable.
Proof: Due to the equivalent of uniform stability and stability between cases of $a=0$ and $a=-\infty$, we only prove here the case of $a=0$. Suppose that (10) is uniformly asymptotically stable. Pick numbers $K$ and $T_{m}$ such that if $\|\phi\|_{t_{0}} \leq 1$, then $\left|x\left(t, t_{0}, \phi\right)\right| \leq K$ for all $t \geq t_{0}$ and $\left|x\left(t+T_{m}+t_{0}, t_{0}, \phi\right)\right|<1 / m$ for all $t \geq 0$. Let $g(t)$ be a continuous, nonincreasing, positive function such that $g(t)=K$ on $0 \leq t \leq T_{1}$, and $g\left(T_{m}\right)=1 /(m-1)$ for $m=$ $2,3,4, \ldots$ Then $\left|x\left(t+t_{0}, t_{0}, \phi\right)\right| \leq g(t) \rightarrow 0$ as $t \rightarrow \infty$, whenever $t_{0} \geq 0$ and $\|\phi\|_{t_{0}} \leq 1$. For this $g(t)$ there exists a function $G(y) \in$ $C^{1}\left(R^{+}\right), G(y)>0, G^{\prime}(y)>0$ for all $y>0, G(0)=G^{\prime}(0)=$ $0, G^{\prime}(y)$ is increasing in $y$ and for constant $C>0$, integrals

$$
\int_{0}^{\infty} G(C g(s)) d s \text { and } \int_{0}^{\infty} G^{\prime}(C g(s)) d s
$$

are finite. Define a Liapunov functional [10]

$$
V(t, \phi)=\int_{0}^{\infty} G\left(\left|x\left(s+t, t_{0}, \phi\right)\right|\right) d s
$$

where $x\left(t, t_{0}, \phi\right)$ is the unique solution of (10) with initial value pair $\left(t_{0}, \phi\right) . V(t, \phi)$ has following properties:

1) $V(t, \phi)$ is locally Lipschitz continuous in $\phi$;

2) $V(t, 0) \equiv 0$, for all $t \geq 0$

3) $V(t, \phi) \geq w_{0}(|\phi(t)|)$;

4) $\dot{V}(t, \phi) \leq-w_{1}(|\phi(t)|)$;

where $w_{0}(y)$ and $w_{1}(y)$ are positive define continuous functions and $\dot{V}(t, \phi)=\limsup _{h \rightarrow 0+}\left\{V\left(t_{0}+h, x\left(\cdot, t_{0}, \phi\right)\right)-V\left(t_{0}, \phi\right)\right\} / h$ is the upper-right Dini derivative of $V(t, \phi)$ with respect to (10).

We now use the same Liapunov functional to control the nonlinear (9).

Let $\psi(t)=u\left(t, t_{0}, \phi\right)$ be any solution of (9). Then

$$
\left.\dot{V}\left(t+t_{0}, \psi\right)\right|_{(9)} \leq\left.\dot{V}\left(t+t_{0}, \psi\right)\right|_{(10)}+C\left|P\left(t+t_{0}, \psi\right)\right|
$$

where $C$ be the Lipcshitz constant for $V(t, \phi)$ and

$$
P(t, u)=F\left(\int_{0}^{t} B(t-s) u(s) d s\right)-\int_{0}^{t} L B(t-s) u(s) d s .
$$

Since $F(\cdot) \in \mathcal{L}$ and $B(\cdot) \in L^{1}(0, \infty)$, we have that $P(t, \cdot) \in \mathcal{L}$. Then the uniform stability of (9) follows from (13) and the proof is similar to that in [10].

In order to prove the global uniform asymptotic stability of (9), we need to show $\psi(t)$ uniformly tends to zero as $t \rightarrow \infty$.

Suppose that $F(\cdot)$ in (9) satisfies global Lipschitz continuality condition (4). Then for $u_{i}(t) \neq 0$ we have

$$
\begin{aligned}
& \frac{d\left|u_{i}(t)\right|}{d t} \leq a_{i i}\left|u_{i}(t)\right|+\sum_{j \neq i}\left|a_{i j}\right|\left|u_{j}(t)\right| \\
& +\sum_{j=1}^{n} \ell_{j} \int_{a}^{t}\left|b_{i j}(t-s)\right|\left|u_{j}(s)\right| d s
\end{aligned}
$$

for $i=1,2,3, \ldots, n$, where $L=\operatorname{diag}\left(\ell_{1}, \ldots, \ell_{n}\right)$ and the upper right Dini derivative is used.

Using (12), we define a Liapunov functional

$$
V(t, \psi)=\int_{0}^{\infty} G(y(s+t)) d s
$$

with

$$
y(t)=\sum_{i=1}^{n}\left|\psi_{i}(t)\right|+\sum_{j=1}^{n} \ell_{j} \int_{0}^{\infty}\left|b_{i j}(u)\right|\left(\int_{t-u}^{t}\left|\psi_{j}(r)\right| d r\right) d u .
$$


By calculating the upper-right Dini derivative of $V(t, \psi)$ with respect to (9), we have

$$
\begin{aligned}
\left.\dot{V}(t, \psi)\right|_{(9)} & =\int_{0}^{\infty} G^{\prime}(y) \frac{d y(t)}{d t} \\
& \leq-\int_{0}^{\infty} G^{\prime}(y) \sum_{i=1}^{n} \lambda_{i}\left|\psi_{i}(t)\right| d s
\end{aligned}
$$

where $\lambda_{i}=\left|a_{i i}\right|-\sum_{j \neq i}\left|a_{i j}\right|+\ell_{i} \sum_{j=1}^{n} \beta_{i j}^{+}$. It follows from (18) and Theorem 1 that $V(t, \psi)$ is bounded. As $G\left(\sum_{i=1}^{n}\left|\psi_{i}(t)\right|\right) \leq G(y(t))$, so, $G\left(\sum_{i=1}^{n}\left|\psi_{i}(t)\right|\right) \in L^{1}(0, \infty)$. From the definition of $G(\cdot)$, we have $\left|\psi_{i}(t)\right|$ uniformly tends to zero as $t \rightarrow \infty$.

Suppose that $F(\cdot) \in \mathcal{G L}$ in (9) and the condition (ii) in Theorem 1 is satisfied, then for $u_{i}(t) \neq 0$ we have

$$
\begin{aligned}
\frac{d\left|u_{i}(t)\right|}{d t} \leq & a_{i i}\left|u_{i}(t)\right|+\sum_{j \neq i}\left|a_{i j}\right|\left|u_{j}(t)\right| \\
& +\ell_{i} \int_{a}^{t} b_{i i}(t-s)\left|u_{i}(s)\right| d s \\
& +\sum_{j \neq i} \ell_{j} \int_{a}^{t}\left|b_{i j}(t-s)\right|\left|u_{j}(s)\right| d s .
\end{aligned}
$$

A simple modification of Liapunov functional (16) will work in the same way, and we omit the details here. As for the conditions in Proposition 1 , we may achieve the conclusion just by multiplying $p_{i}$ to $y(t)$ in (17) and to follow the same arguments. The proof of the case $a=-\infty$ can be repeated step by step as above.

Form Proposition 2, the sufficient conditions for the stability of a linear Volterra system in Theorem 1 and Proposition 1 can be used to determine the global stability of linear system (10), and through it to $(9)$.

\section{RECURRENT NEURAL NETWORKS}

In this section, we will apply the results from previous section to study the global uniform asymptotic stability of RNNs.

As indicated in the previous section, there is no difference for $a=0$ and $a=-\infty$ on the stability of linear Volterra systems, except that the stability of case $a=-\infty$ is equivalent to the uniform stability of case $a=0$. In order to compare the existing results for the RNNs, in the following, we only consider (1) with $a=-\infty$ and the results will also be valid for other case. If the results apply to (2), we have assumed $u=0$, and no confusion should arise.

If $F \in \mathcal{L}$ in (1), by applying Theorem 1, Propositions 1 and 2, we have the following results.

Corollary 1: The trivial solution of (1) and (2) is globally asymptotically stable if

$$
d_{i} \geq \sum_{j=1}^{n}\left|b_{i j}\right| \ell_{j}, \quad i=1,2, \ldots, n .
$$

Proof: Taking $K_{i i}(s)=\delta(s)$ and verifying the condition (i) of Theorem 1.

Corollary 2: The trivial solution of (1) and (2) is globally asymptotically stable if $D-\left(\left|b_{i j}\right|\right) L$ is an $M$-matrix, here $L=\operatorname{diag}\left(\ell_{1}, \ldots, \ell_{n}\right)$.

Corollary 3: [7]: The trivial solution of systems (1) and (2) are globally asymptotically stable if $d_{i}>0$ and there exist $p_{i}>0,(i=$ $1,2, \ldots, n)$ such that

or

$$
d_{i} p_{i}>\sum_{j}^{n}\left|b_{i j}\right| \ell_{j} p_{j}
$$

$$
p_{j} d_{j}>\sum_{i}^{n} p_{i}\left|b_{i j}\right| \ell_{i}
$$

Remark 1: The condition which is similar to Corollary 1 has been seen in the Theorem 2 in [4] and many people have generated it by using $M$-matrix. The results of Corollaries 2 and 3 are the same condition as Theorem 4 in [7] for delay-free system and Theorem 1 in [2] and basically are the same as Theorem 5 in [13], the condition (H2) in [3] and conditions in [15]. We notice that some of these conditions also guarantee the global exponential stability (GES) ([7], [11], [15]) and believe that the uniform asymptotic stability of (5) is of GES if there exists $\lambda>0$, such that $e^{\lambda t}|B(t)| \in L^{1}\left(R^{+}\right)$.

If $F \in \mathcal{G} \mathcal{L}$ in (1), let $E(T)=\int_{0}^{\infty} t\left|k_{i i}(t)\right| d t$ denote the expectation value of $t$ with absolute of synapse filter $k_{i i}(t)$, then applying Theorem 1, Propositions 1, we have the following results.

Corollary 4: The trivial solution of (1) is globally asymptotically stable if for $i=1,2, \ldots, n$

$$
D_{i}^{*}=-d_{i}+\ell_{i} b_{i i} \int_{0}^{\infty}\left|k_{i i}(t)\right|<0,
$$

and

$$
E(T) \leq \frac{-D_{i}^{*}-\sum_{j \neq i}\left|b_{i j}\right| \ell_{j}}{\sum_{j=1}^{n}\left|b_{i j}\right| \ell_{j}} .
$$

Proof: Just verifying the condition (ii) of Theorem 1.

Corollary 5: The trivial solution of (2) is globally asymptotically stable if

$$
d_{i}-\ell_{i} b_{i i} \geq \sum_{j \neq i}\left|b_{i j}\right| \ell_{j}, \quad i=1,2, \ldots, n .
$$

Proof: Verifying (22) of Corollary 4 or directly verifying the condition (i) of Proposition 1.

Corollary 6: The trivial solution of

$$
\frac{d x(t)}{d t}=-D x(t)+B F(x(t-\tau))
$$

is globally asymptotically stable if for $i=1,2, \ldots, n$

$$
\tau_{i} \leq \frac{d_{i}-b_{i i} \ell_{i}-\sum_{j \neq i}\left|b_{i j}\right| \ell_{j}}{\sum_{j=1}^{n}\left|b_{i j}\right| \ell_{j}} .
$$

Proof: Taking $K_{i i}(s)=\delta\left(s-\tau_{i}\right)$ and verifying (22) of Corollary 2.

Applying Proposition 1, we have.

Corollary 7: The trivial solution of (2) is globally asymptotically stable if $D-\operatorname{diag}\left(\ell_{1} b_{11} \cdots \ell_{n} b_{n n}\right)-\left(\left|b_{i j}\right|\right)_{i \neq j} L$ is an $M$-matrix.

Corollary 8: The trivial solution of (2) is globally asymptotically stable if $d_{i}>0$ and there exist $p_{i}>0,(i=1,2, \ldots, n)$ such that

or

$$
\left(d_{i}-\ell_{i} b_{i i}\right) p_{i}>\sum_{j \neq i}\left|b_{i j}\right| \ell_{j} p_{j}
$$

$$
p_{j}\left(d_{j}-\ell_{j} b_{j j}\right)>\sum_{i \neq j} p_{i}\left|b_{i j}\right| \ell_{i} .
$$

Remark 2: The conditions in Corollaries 5, 7, and 8 have been seen in many papers for differently defined activation functions ([1], [5]), except that the inequalities (23) here are not strict. Corollary 4, Corollary 6, and the following Corollary 9 are valid for $F \in \mathcal{G L}$ and their conditions are delay-dependent, and have not been seen in the literature by such an explicit and intuitive way.

Consider another popular RNN model

$$
\frac{d x}{d t}=-D x+F(W x+u), \quad x\left(t_{0}\right)=x_{0}
$$

which is equivalent to (2) if $W D=D W$ and $W$ is nonsingular ([6], [7]). Similarly, the generalization of (2) can be written as

$$
\left\{\begin{array}{l}
\frac{d x(t)}{d t}=-D x(t)+F\left(B \int_{a}^{t} K(t-s) x(s) d s\right) \\
x(s)=\phi(s), \quad x \in\left(a, t_{0}\right), \quad t_{0} \geq 0 .
\end{array}\right.
$$


Applying Theorem 1, Propositions 1 and 2, we can derive another set of conditions that are very similar with Corollaries $1-8$ except in the inequalities $\ell$ s are out of the sum sign.

The previous results are based on the fact of $D>0$, the self-regulation of negative feedback of each neuron. Letting $F(x)=\alpha F_{1}(x)+$ $\beta F_{2}(x)$ and choosing appropriate $\delta$ function as delay kernel from (1), we can obtain the delayed cellular neural networks (DCNNs) ([1], [14])

$$
\frac{d x(t)}{d t}=-D x(t)+E_{1} F_{1}(x(t))+E_{2} F_{2}(x(t-\tau))
$$

where the nondelayed items may contribute to neurons self regulation or interactions. We can still apply our results in Section II to compare the neural networks under consideration to some suitable linear systems according to the class of Lipchitz activation functions.

The following example was considered by Cao and Wang [1] without delays. Suppose that $K(\cdot) \in L^{1}\left(R^{+}\right)$. Then we modify this example in the following form

$$
\left\{\begin{array}{l}
\frac{d x_{1}(t)}{d t}=-7 x_{1}(t)+2 f_{1}\left(\int_{-\infty}^{t} k_{1}(t-s) x_{1}(s) d s\right) \\
\quad+8 f_{2}\left(\int_{-\infty}^{t} k_{2}(t-s) x_{2}(s) d s\right) \\
\frac{d x_{2}(t)}{d t}=-11 x_{2}(t)+2 f_{1}\left(\int_{-\infty}^{t} k_{1}(t-s) x_{1}(s) d s\right) \\
\quad-5 f_{2}\left(\int_{-\infty}^{t} k_{2}(t-s) x_{2}(s) d s\right), \\
x_{1}(s)=\phi_{1}(s), x_{2}(s)=\phi_{2}(s), s \in(-\infty, \tau), \tau \geq 0
\end{array}\right.
$$

where $D=\left[\begin{array}{cc}7 & 0 \\ 0 & 11\end{array}\right], B=\left[\begin{array}{cc}2 & 8 \\ 2 & -5\end{array}\right]$ and $f_{i}=-\left|x_{i}\right|(i=1,2)$. Clearly, $f_{i} \in \mathcal{L}$ is unbounded and Lipschitz continuous with the Lipschitz constant $k_{i}=1$. We can check that $D-L\left(\left|b_{i j}\right|\right)_{2 \times 2}=$ $\left[\begin{array}{cc}5 & -8 \\ -2 & 6\end{array}\right]$ is an $M$-matrix. Hence, by Corollary 2 the trivial solution of (29) is globally asymptotically stable.

Now, we further modify the above example to a DCNN model (28) by letting

$$
\begin{aligned}
E_{1} & =\left[\begin{array}{ll}
8 & 0 \\
6 & 2
\end{array}\right] \\
F_{1}(x(t)) & =\left[\begin{array}{l}
\tanh \left(x_{1}(t)\right) \\
\tanh \left(x_{2}(t)\right)
\end{array}\right] \\
E_{2} & =\left[\begin{array}{cc}
-2 & \frac{1}{2} \\
2 & -5
\end{array}\right] \\
F_{2}(x(t-\tau)) & =\left[\begin{array}{l}
\tanh \left(x_{1}\left(t-\tau_{1}\right)\right) \\
\tanh \left(x_{2}\left(t-\tau_{2}\right)\right)
\end{array}\right] .
\end{aligned}
$$

Obviously, $F_{1}, F_{2} \in \mathcal{G} \mathcal{L}$. It is easy to check $D-\left(\left|E_{1}\right|\right) K-\left(\left|E_{2}\right|\right) L=$ $\left[\begin{array}{cc}-3 & -(1 / 2) \\ -8 & 4\end{array}\right]$ is not an $M$-matrix, so the Theorem 3 in [1] cannot be applied for (28), where $k=L=\operatorname{diag}(1,1)$. By using Dini derivative and the properties of function class $\mathcal{G L}$, we have

$$
\left\{\begin{array}{l}
\frac{d\left|x_{1}(t)\right|}{d t} \leq x_{1}(t)-2 x_{1}\left(t-\tau_{1}\right)+\frac{1}{2}\left|x_{2}\left(t-\tau_{2}\right)\right| \\
\frac{d\left|x_{2}(t)\right|}{d t} \leq 6\left|x_{1}(t)\right|-9 x_{2}(t)-2\left|x_{1}\left(t-\tau_{1}\right)\right|-5 x_{2}\left(t-\tau_{2}\right) .
\end{array}\right.
$$

Following from Proposition 2 and checking the condition (ii) of the Theorem 2, we can claim that when

$$
\left\{\begin{array}{l}
\tau_{1} \leq \frac{1}{7} \\
\tau_{2} \leq \frac{3}{11}
\end{array}\right.
$$

the DCNN system is globally asymptotically stable.

For some cases, one can use a special distribution function to describe the special delay effects approximately. Other useful delay kernels are given by Gamma density function

$$
k_{i j}{ }^{(m)}(s)=\frac{\gamma_{i j}{ }^{m}}{\Gamma(m)} s^{m-1} e^{-\gamma_{i j} s}, \quad m>0, \quad s>0
$$

or its linear combinations, where

$$
\Gamma(m)=\int_{0}^{\infty} e^{-t} t^{m-1} d t, \quad m>0
$$

is the $\Gamma$-function and $\gamma_{i j}$ are real constants. From Corollary 2, we have.

Corollary 9: Let delay kernel be in the form of (32). Then the trivial solution of (1) is globally asymptotically stable if for all $i=1,2, \ldots$,

$$
\frac{m}{\gamma_{i i}} \leq \frac{d_{i}-b_{i i} \ell_{i}-\sum_{j \neq i}\left|b_{i j}\right| \ell_{j}}{\sum_{j=1}^{n}\left|b_{i j}\right| \ell_{j}} .
$$

\section{CONCLUDING REMARKS}

Sufficient conditions have been derived for the GAS of the equilibrium of recurrent neural networks with distributed delay by applying the theory on Volterra integro-differential equations. The conditions, which lead to GAS, also guarantee the uniqueness of the equilibrium. Thus, we can avoid the difficulties on proving the uniqueness of the equilibrium, which forms the underlying basis for ad hoc constructing of special Liapunov functions or functionals.

\section{ACKNOWLEDGMENT}

The authors would like to thank the Associate Editor and the anonymous reviewers for their valuable comments and constructive suggestions. The authors also thank Professor J. Sun at Tongji University, China, for his reading and suggestion on the final version of the letter.

\section{REFERENCES}

[1] J. Cao and J. Wang, "Global asymptotic stability of a general class of recurrent neural networks with time-varying delays," IEEE Trans. Circuits Syst. I, Fundam. Theory Appl., vol. 50, no. 1, pp. 34-44, Jan. 2003.

[2] Y. Chen, "Global stability of networks with distributed delays," Neural Netw., vol. 15, no. 7, pp. 867-871, Sept. 2002.

[3] C. Feng and R. Plamondon, "On the stability analysis of delayed neural networks systems," Neural Netw., vol. 14, no. 9, pp. 1181-1188, Nov. 2001.

[4] K. Gopalsamy and X. He, "Delay-independent stability in bidirectional associative memory networks," IEEE Trans. Neural Netw., vol. 5, no. 6, pp. 998-1002, Nov. 1994.

[5] Z. Guan, G. Chen, and Y. Qin, "On equilibria, stability, and instability of Hopfield networks," IEEE Trans. Neural Netw., vol. 11, no. 2, pp. 534-540, Mar. 2000.

[6] S. Haykin, Neural Networks, A Comprehensive Fundation, 2nd ed. Englewood Cliffs, NJ: Prentice-Hall, 1999.

[7] S. Hu and J. Wang, "Global stability of a class of continuous-time recurrent neural networks," IEEE Trans. Circuits Syst. I, Fundam. Theory Appl., vol. 49, no. 9, pp. 1334-1347, Sep. 2002.

[8] F. Izsak, "An existence theorem for Volterra integrodifferential equations with infinite delay," Electron. J. Diff. Equ., vol. 2003, no. 4, pp. 1-9, Jan. 2003. URL:

[9] G. S. Jodan, "Asymptotic stability of a class of integrodifferential systems," J. Differ. Equ., vol. 31, no. 3, pp. 359-365, Mar. 1979.

[10] R. K. Miller, "Asymptotic properties of linear volterra integrodiffererntial equations," J. Differ. Equ., vol. 10, no. 3, pp. 485-506, Nov. 1971.

[11] L. Rong, W. Lu, and T. Chen, "Globa exponential stability in Hopfield and bidirectional associative memory neural networks with time delays," Chinese Anna. Math., vol. 25, no. 2, pp. 255-262, Feb. 2004.

[12] J. Zhang and Y. Yang, "Global stability analysis of bidirectional associative memory neural networks with time delay," Int. J. Circuit Theor. Appl., vol. 29, no. 2, pp. 185-196, Mar./Apr. 2001.

[13] J. Zhang and X. Jin, "Global stability analysis in delayed Hopfied neural network models," Neural Netw., vol. 13, no. 7, pp. 745-753, Sep. 2000.

[14] Q. Zhang, R. Ma, C. Wang, and J. Xu, "On the global stability of delayed neural networks," IEEE Trans. Autom. Control, vol. 48, no. 5, pp. 794-797, May 2003.

[15] Q. Zhang, X. Wei, and J. Xu, "Global exponential stability of Hopfield nueral networks with continuously distributeed delays," Phys. Lett. A, vol. 315 , no. 6 , pp. 431-436, Sep. 2003. 